\title{
Full Stress Tensor Measurement by Photoelasticity in Silicon
}

\author{
Markus Stoehr ${ }^{1}$, Gerald Gerlach², Thomas Härtling2,3, Stephan Schoenfelder ${ }^{1}$ \\ 1 University of Applied Sciences Leipzig, Faculty of Engineering Sciences \\ ${ }^{2}$ Technische Universität Dresden, Institut für Festkörperelektronik \\ ${ }^{3}$ Fraunhofer Institute for Ceramic Technologies and Systems IKTS \\ markus.stoehr@htwk-leipzig.de
}

\begin{abstract}
Summary:
Photoelasticity offers a promising measurement tool for the in-line measurement of semiconductor materials such as silicon. Photoelasticity is a contactless, optical and full-field measurement method based on stress-induced birefringence. However, it is in principle only capable of measuring stress differences and therefore not able to determine the quantitative stress state inside a material. In this work a method is presented to separate this stress difference using modified equations of the mechanical equilibrium in conjunction with the Finite-Difference-Method. This method takes into account the anisotropic photoelastic law for mono-crystalline silicon and enables a full separation of the mechanical stresses with a single measurement.
\end{abstract}

Keywords: photoelasticity, stress measurement method, full-field, contactless, semiconductors

\section{Introduction}

Photoelasticity is regarded a promising measurement principle to characterize stress fields inside thin substrates. It is based on stress-induced birefringence, which causes a material to split an incoming light wave into two light waves each with different coefficients of refraction. In general, only the stress difference $\left(\sigma_{11}-\sigma_{22}\right)$ and the shear stresses $\sigma_{12}$ can directly be measured with this. However, a full stress tensor information gives the often more desirable full image of the stress state inside a material or component.

In this work a method is developed to separate the full stress tensor information from a single photoelastic measurement. Since silicon shows a mechanically and photo-elastically anisotropic behavior, first the photo-elastical law for monocrystalline silicon is developed. A general approach and the explicit stress-optical relationship for several crystalline orientations are derived. With those, the stress differences and the shear stresses can be measured. Second, a method is developed to separate the stress difference into the single stress components by solving modified equations of the mechanical equilibrium. These $2^{\text {nd }}-$ order partial differential equations are approximated by the Finite-Difference-Method. Applying FDM to the differential equations and using known initial values, the stress difference $\left(\sigma_{11}-\sigma_{22}\right)$ can further be separated into the single stress components $\sigma_{11}$ and $\sigma_{22}$.
This method is demonstrated for the full stress tensor determination of a silicon wafer under diametrical load and for the measurement of residual stresses in a multi-crystalline silicon slab.

\section{Methods}

Photoelasticity can be described using a phenomenological approach suggested by Pockels $[1,2]$. This model states that mechanical stresses lead to a change of the impermeability tensor $B_{i j}$ which describes the optical properties. The change of impermeability is proportional to the stress tensor $\sigma_{i j}$ by the stress-optical tensor $\pi_{i j k l}$ and adds to the unstressed impermeability $B_{i j}^{O}$ :

$$
B_{i j}=B_{i j}^{o}+\pi_{\mathrm{ijkl}} \sigma_{k l}
$$

The change of impermeability depends also on the crystalline orientation and the load direction. Therefore, the stress-optical tensor has to be transformed accordingly.

In this work, a general stress-optical relation and explicit relations for a (100), (110) and (111) crystalline orientation are developed. The necessary stress-optical material parameters $\left(\pi_{11}-\right.$ $\pi_{12}$ ) and $\pi_{44}$ are determined using a (100) silicon wafer under diametrical load inside a grey-field polariscope.

With this, the stress difference $\left(\sigma_{11}-\sigma_{22}\right)$ and the shear stress $\sigma_{12}$ can be measured. To further separate this difference into its single stress 

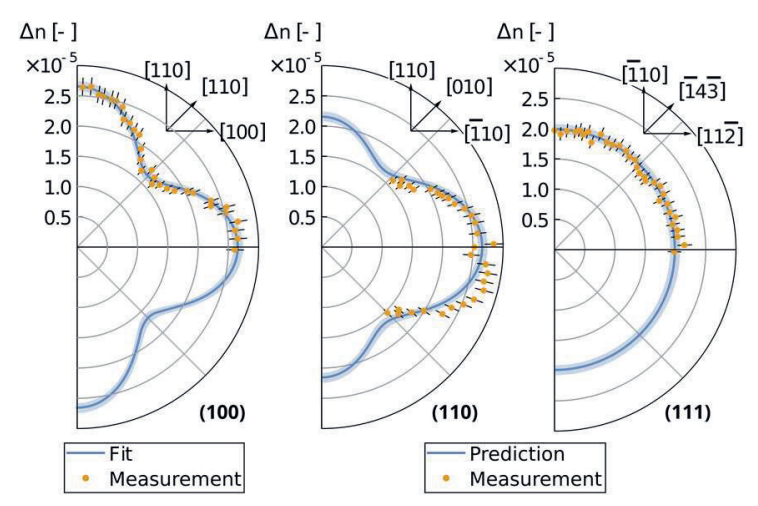

Fig. 1: Stress-optical relations (blue) for a (100), (110) and (111) orientation of silicon with measurement results (yellow)

components a modified equation of the mechanical equilibrium is used [3]:

$$
\frac{\partial^{2} \sigma_{11}+\sigma_{22}}{\partial x_{1}^{2}}+\frac{\partial^{2} \sigma_{11}-\sigma_{22}}{\partial x_{1}^{2}}+2 \frac{\partial^{2} \sigma_{12}}{\partial x_{1} \partial x_{2}}=0 .
$$

The Finite-Difference-Method is applied to this equation in a central difference scheme to approximate the derivations over a discretized domain. This gives a set of equations for each discretized point that can be solved to obtain the single stress components $\sigma_{11}$ and $\sigma_{22}$. For this, initial values are necessary that can be taken from free edges of the measured component.

\section{Results}

The stress-optical material parameters are measured using a (100) silicon wafer under diametrical load as $\left(\pi_{11}-\pi_{12}\right)=(12.4 \pm 0.3)$. $10^{-7} \mathrm{MPa}^{-1}$ and $\pi_{44}=(7.7 \pm 0.3) \cdot 10^{-7} \mathrm{MPa}^{-1}$. With those material parameters and with the respective theoretical derivation a prediction is made for the stress-optical law for the (110) and (111) orientation. This prediction follows closely the measurement results of the respective crystalline orientations, as shown in fig. 1.

The separation of the stress difference $\left(\sigma_{11}-\right.$ $\sigma_{22}$ ) into the single stress components was tested for the (100) silicon wafer under diametrical load. The stress components were found to be in good agreement with a Finite-Element-Analysis of the same test, as can be seen in fig. 2 .

Additionally, the method was applied to a silicon slab that was cut from a multi-crystalline silicon ingot. The stress-optical law was averaged for the various orientations of the crystallites. With that, the determined stresses are very similar to
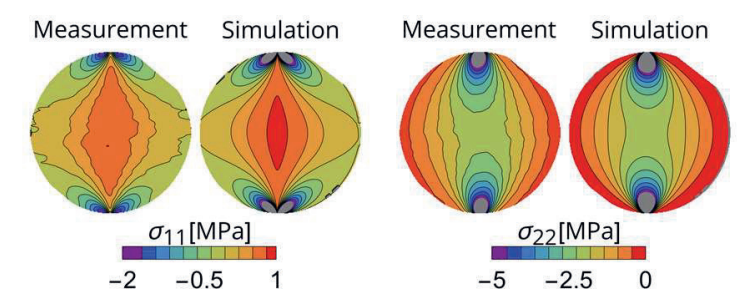

Fig. 2: Stress components for a (100) silicon wafer under diametrical load

experimental works by Bär et al. [4] using the stress-relation method.

\section{Discussion and Conclusion}

In this work a method is presented that enables a full-field quantitative stress characterization of thin silicon substrates with a single photo-elastical measurement.

The method was tested and validated using a (100) silicon wafer under diametrical load. The determined stresses correspond well to the stresses simulated by means of FEM. Furthermore, this method was applied to the measurement of residual stresses in a multi-crystalline silicon slab where it showed a good agreement to previous experimental works. However, the experimental effort for photo-elastical measurements is considerably lower compared to the laborious stress-relaxation method used previously by Bär et al.

While this approach was demonstrated for silicon, it can be applied to a variety of different semiconductor materials. Therefore, this method presents interesting opportunities for the application to in-line measurements in production or for academic purposes.

\section{References}

[1] K. Neumann, Die Gesetze der Doppelbrechung des Lichts in comprimirten oder ungleichförmig erwärmten unkrystallinischen Körpern, Annalen der Physik und Chemie, 54, 12 , 449 - 476, 1841

[2] F. Pockels, Ueber den Einfluss elastischer Deformationen, speciell einseitigen Druckes, auf das optische Verhalten krystallinischer Körper, Annalen der Physik, 273, 6, 269 - 305, 1889

[3] L. J. Segerlind, Stress-difference elasticity and its application to photomechanics, Experimental Mechanics, 11, 440 - 445, 1971

[4] T. Bähr et al., Residual stresses in multicrystalline silicon, In: 27th European Photovoltaic Solar Energy Conference and Exhibition; 642-646 